\title{
Detection of negative ions in glow discharge mass spectrometry for analysis of solid specimens
}

\author{
Stela Canulescu • Igor S. Molchan • C. Tauziede • \\ Agnes Tempez • J. A. Whitby • George E. Thompson • \\ Peter Skeldon • P. Chapon • Johann Michler
}

Received: 18 September 2009 /Revised: 30 November 2009/Accepted: 30 November 2009/Published online: 24 December 2009

(C) Springer-Verlag 2009

\begin{abstract}
A new method is presented for elemental and molecular analysis of halogen-containing samples by glow discharge time-of-flight mass spectrometry, consisting of detection of negative ions from a pulsed RF glow discharge in argon. Analyte signals are mainly extracted from the afterglow regime of the discharge, where the cross section for electron attachment increases. The formation of negative ions from sputtering of metals and metal oxides is compared with that for positive ions. It is shown that the negative ion signals of $\mathrm{F}^{-}$and $\mathrm{TaO}_{2} \mathrm{~F}^{-}$are enhanced relative to positive ion signals and can be used to study the distribution of a tantalum fluoride layer within the anodized tantala layer. Further, comparison is made with data obtained using glow-discharge optical emission spectroscopy, where elemental fluorine can only be detected using a neon plasma. The ionization mechanisms responsible for the formation of negative ions in glow discharge time-offlight mass spectrometry are briefly discussed.
\end{abstract}

Keywords RF glow discharge mass spectrometry.

Negative ions · Fluorine ions · Pulsed discharge .

S. Canulescu $(\bowtie) \cdot J$. A. Whitby $\cdot$ J. Michler

Laboratory for Mechanics of Materials and Nanostructures,

EMPA, Swiss Federal Laboratories for Materials Testing

and Research, Feuerwerkerstrasse 39,

Thun 3602, Switzerland

e-mail: stela.canulescu@gmail.com

e-mail: stela.canulescu@empa.ch

I. S. Molchan • G. E. Thompson • P. Skeldon

Corrosion and Protection Centre, School of Materials,

The University of Manchester, P. O. Box 88,

Manchester M60 1QD, UK

C. Tauziede $\cdot$ A. Tempez $\cdot$ P. Chapon

HORIBA Jobin Yvon SAS, 91160 Longjumeau, France
Anodic tantala $\cdot$ Mass spectrometry/ICP-MS · Thin films · (XPS|XRF|EDX)

\section{Introduction}

Recent achievements in the field of materials science have led to significant progress in spectrochemical methods for analytical applications [1, 2]. For example, inductively coupled plasma-mass spectrometry (ICP-MS) is an established technique for solution analysis [3], and secondary neutral mass spectrometry is a developing tool for quantitative surface analysis with high lateral resolution [4]. Glow discharge mass spectrometry (GDMS) is a powerful analytical method for the direct determination of trace elements in bulk solids down to pbb levels [5], while the newest developed prototypes, i.e., glow discharge time-offlight mass spectrometry (GD-TOFMS), are also used to offer rapid quantitative depth profiling of ultra-thin $(\mathrm{nm})$ and thin $(\mu \mathrm{m})$ coatings [6-8]. Progress in the development of instrumentation utilizing pulsed RF glow discharges has enabled the analysis of both electrically conducting and non-conducting materials. Muñiz et al. have demonstrated the analytical capabilities of a pulsed RF-GD-TOFMS system for analysis of multi-nanolayers deposited on glass [9]. Recent advances in GD-TOFMS have been focused on important, new analytical applications, i.e., rapid molecular depth profiling of polymers [10].

Analytical GD devices commonly use argon as the carrier gas for the atomization and ionization of the analytes. In pulsed GD, collisions between sputtered atoms and metastable argon atoms are considered to be responsible for the formation of positive ions upon the termination of the applied power ("afterglow") [11, 12]. The afterglow 
regime, rich in argon metastables, favors the formation of positive ions through Penning ionization (PI) processes [13]. The argon metastable atoms, with high energy levels (e.g., 11.55 or $11.72 \mathrm{eV}$ ), can ionize most of the elements in the periodic table. Relatively few elements cannot be ionized by $\mathrm{PI}$ in a glow discharge in argon, e.g., $\mathrm{H}, \mathrm{N}, \mathrm{O}, \mathrm{F}, \mathrm{CI}$, and $\mathrm{Br}$.

In GDMS, positive ion detection has yielded excellent analytical signals for most elements, but there are several limitations of the technique. The presence of isobaric interferences limits the detection sensitivity for some elements. For example, ${ }^{19} \mathrm{~F}^{+}$has interference from ${ }^{19} \mathrm{H}_{3} \mathrm{O}^{+},{ }^{40} \mathrm{Ca}^{+}$from ${ }^{40} \mathrm{Ar}^{+}$, and ${ }^{80} \mathrm{Se}^{+}$from ${ }^{80} \mathrm{Ar}_{2}{ }^{+}$(required resolving power $m / \Delta m \sim 9,000$ ).

The analytical utility of negative ions has been explored in ICP-MS systems, where the halogens have been detected with good sensitivities [14]. There have been relatively few studies on detection of negative ions from a GD in argon [15]. Recent trends in GD-TOFMS have shown the potential use of negative ions for analytical applications [16]. An enhancement in the afterglow by at least three orders of magnitude for $\mathrm{F}^{-}$was reported from sputtered PTFE polymer fragments.

In order to evaluate the performance of newly developed instrumentation, samples with well-known characteristics are required. An ideal material for testing and evaluation is anodic tantala [17]. Anodic tantala films may be formed in a very wide range of electrolytes, allowing incorporation of appropriate species. The mobility of many species, including fluorine, during formation of anodic tantala films is well known that precise prediction of their distribution within the film is possible [18].

Here, anodic tantala samples, specially produced with known distributions of fluorine species, have been examined using a GD-TOFMS instrument in the negative ion mode. The presence of molecular ions containing fluorine provides a clear evidence of enhanced plasma chemistry and enables differentiation between the relatively pure tantala layers and the tantalum layer deliberately doped with fluorine.

\section{Experimental}

Instrumentation

The GD-TOFMS instrument consists of a high-resolution time-of-flight mass analyzer (Tofwerk AG) coupled to a prototype RF-GD ion source [16]. In addition, an interface system has been implemented to improve transmission and to allow selected mass blanking (e.g., to suppress large signals from argon in order to improve the performance of the instrument). The GD source is a modified Grimm-type source with an inner diameter of $4 \mathrm{~mm}$ and an extended flow tube of $16 \mathrm{~mm}$, which guides ions from the source to the sampler, i.e., a fast flow source [19]. It operates using argon as carrier gas (purity $99.9999 \%$ ) in a typical working pressure in the source in between 200 and $500 \mathrm{~Pa}$. The carrier gas is additionally purified using a Vici P100-1 inert gas purifier. An argon flow of about $0.2 \mathrm{l} / \mathrm{min}$ enhances the transport of ions from the sample to the sampler. Ions generated in the GD source are extracted through a sampling orifice of $340 \mu \mathrm{m}$ diameter. The RF generator (13.56 MHz) and matching box are from Horiba Jobin Yvon. The RF power is supplied to the back of the sample using a water-cooled cylindrical coupler. The RF generator can be operated in pulsed mode with a frequency ranging from 0.1 to $10000 \mathrm{~Hz}$ and a duty cycle in a range from 0.1 to 0.5 . For time-resolved experiments, the RF generator can be operated at a minimum pulsed width of $500 \mu \mathrm{s}$ and 0.1 duty cycle. During the measurements, the RF coupler (and thus the specimen) is cooled to about $7^{\circ} \mathrm{C}$.

The TOF analyzer is an orthogonal extraction reflectron using a MCP detector. It can operate at a maximum extraction frequency of $100 \mathrm{kHz}$ (corresponding to a mass range up to $100 \mathrm{Th}$ ). This high time resolution means that many discrete mass spectra can be acquired over the length of an RF pulse and the subsequent afterglow. In the data reduction process, signals may be chosen from any part of the powered phase or afterglow as appropriate. For convenience, several RF pulses are averaged.

For analysis of metals, a RF pulse of 2 ms was followed by an off-pulse of $6 \mathrm{~ms}$. The discharge conditions of $350 \mathrm{~Pa}$ argon pressure and $15 \mathrm{~W}$ RF power (pressure and power are those indicated by the RF bay control software from Jobin Yvon) were found to yield to large signal intensities. The TOFMS was operated with an extraction frequency of $15 \mathrm{kHz}$ to give a mass range of about 1,400 Th.

The discharge conditions for analysis of the tantalum layer are a compromise between sensitivity and depth resolution. GD-TOFMS depth profiling of tantala layers was carried out using pulse widths varying between 0.5 and $2 \mathrm{~ms}, 300 \mathrm{~Pa}$ argon pressure and $60 \mathrm{~W}$ RF power.

The mass analyzer has bi-polar power supplies to allow either positive or negative ion detection mode operation. For the negative ion mode, the polarity of all ion optic potentials is switched. Switching between positive and negative ion detection modes is relatively fast $(1 \mathrm{~min})$. In this paper, only results from negative ion mode operation are shown. Each negative mass spectrum is calibrated using the ${ }^{16} \mathrm{O}^{-}$and ${ }^{79} \mathrm{Br}^{-}$peaks that are always present in the background spectrum. (The origin of the bromine is unknown but it is a persistent signal. The oxygen is believed to arise from air and water desorbed from the surface of the sample and the source.)

Additionally, the elemental depth distributions of the anodized tantala layers were examined by GD-OES, using a GD-Profiler 2 (Horiba Jobin Yvon). A 4-mm-diameter copper anode and pure (99.9998\%) neon gas were used. 
Neon is required in GD-OES to excite emission from fluorine. The emission responses from the excited sputtered elements were detected with a polychromator of focal length $500 \mathrm{~mm}$ with 42 optical windows. The emission lines used were $156.149 \mathrm{~nm}$ for $\mathrm{C}, 324.75 \mathrm{~nm}$ for $\mathrm{Cu}$, 121.56 for $\mathrm{H}, 130.21 \mathrm{~nm}$ for $\mathrm{O}$, and $362.66 \mathrm{~nm}$ for Ta. The $F$ signal was recorded using the $685.611 \mathrm{~nm}$ line on the monochromator. Elemental depth profiling was carried out at $950 \mathrm{~Pa}$ and $60 \mathrm{~W}$ with a data acquisition time of $0.01 \mathrm{~s}$ for the first $30 \mathrm{~s}$ then of $0.1 \mathrm{~s}$. Prior to depth profiling measurement, a sacrificial Si layer was sputtered for $60 \mathrm{~s}$ for cleaning purposes [20]. For both steps (Si pre-sputtering and specimen analysis), the neon gas flow was run for $30 \mathrm{~s}$ before commencing the plasma.

\section{Specimen preparation}

Anodic tantala films were prepared by anodizing a tantalum layer formed by magnetron sputtering on a $0.3 \mathrm{~mm}$ thickness aluminum foil substrate supporting an anodic alumina film. Initially, the $99.99 \%$ purity aluminum foil was electropolished in a perchloric acid/ethanol (20/ 80 vol.\%) mixture at temperatures less than $10^{\circ} \mathrm{C}$ for $5 \mathrm{~min}$. Individual aluminum substrates were then anodized in $0.1 \mathrm{M}$ ammonium pentaborate at $5 \mathrm{~mA} / \mathrm{cm}^{2}$ to a voltage of $250 \mathrm{~V}$, forming a barrier-type anodic film of $300 \mathrm{~nm}$ thickness. This procedure was utilized to generate highly flat surfaces that provide good adhesion of the subsequently deposited tantalum films. Sputter deposition of tantalum was performed using a 99.9\% purity tantalum target for $2 \mathrm{~h}$ at a constant current of $200 \mathrm{~mA}$, with a residual gas pressure of $4 \times 10^{-7} \mathrm{mbar}$ and a total pressure of $5 \times 10^{-3}$ mbar of purified argon. Deposited tantalum layers were anodized in individual $0.1 \mathrm{M}$ ammonium pentaborate (APB) and $1 \mathrm{M}$ ammonium fluoride (AF) electrolytes at $1 \mathrm{~mA} / \mathrm{cm}^{2}$. Further, sequential anodizing in both electrolytes enabled formation of anodic tantala films with controlled distributions of incorporated fluoride species. The following three sample types were prepared: sample $\mathrm{F} 100 \mathrm{~V}$, formed by anodizing in AF to $100 \mathrm{~V}$; sample B80V-F100V, formed by anodizing in APB to $80 \mathrm{~V}$ followed by re-anodizing in AF to $100 \mathrm{~V}$; sample B90VF100V-B120V, formed by anodizing in APB to $90 \mathrm{~V}$, followed by re-anodizing in $\mathrm{AF}$ to $100 \mathrm{~V}$ and final re-anodizing in APB to $120 \mathrm{~V}$.

\section{Results and discussions}

Formation, transport, and sampling of negative ions

Argon plasmas are normally strongly electropositive, meaning that most ions are positive, and the negative charge is carried almost entirely by electrons. Under these circumstances, due to the higher mobility of electrons than positive ions, an anode sheath forms at grounded surfaces. This sheath has a potential gradient that tends to accelerate positive ions through the sheath, but decelerates negatively charged species. Due to the presence of this sheath and the low number of negative ions present, mass spectrometric observations of argon plasma through a grounded sampling orifice are not expected to yield significant signals for negative ions. This statement is complicated by the possibility of negative ions forming within the anode sheath, in the flowing afterglow plasma downstream of the sampling orifice, or by simply having gained sufficient energy in the cathode sheath to penetrate the anode sheath (true also for RF discharges) [21]. For a pulsed discharge, after the power is removed, the electron temperature falls rapidly, and the electron number density will also drop (not necessarily monotonically). These two effects promote the formation of negative ions and their transport to a sampling orifice, thus resulting in a large increase in signal $[16,22]$.

The main mechanisms responsible for the negative ions formation in low pressure discharges are electron attachment, dissociative electron attachment, ion pair formation, and charge transfer [21].

\section{Advantages and disadvantages of negative ionization}

Advantages of negative ion detection include simpler background spectra and different (often less) fragmentation of molecular species than evident for electron impact or Penning ionization. In the present study, with a pulsed glow discharge, a very large increase in sensitivity for halogens and other species with high electron affinities is evident. A disadvantage is that the cross sections for electron attachment can vary dramatically for different molecules or molecular radicals, hindering ready interpretation of the resulting mass spectrum from a mixture of species.

Negative ions from sputtered metals and metal oxides

Bentz et al. [15] have published the first report of detecting negative ions from a glow discharge in argon by mass spectrometry, where only $\mathrm{Au}^{-}$ions were detected. Efficient mass spectrometric sampling can be achieved by pulsing the plasma and collecting ions in the afterglow.

Figure 1a illustrates a negative mass spectrum sampled from the integration of the afterglow region of the pulse profile between 2 and $5 \mathrm{~ms}$. The negative pulse profiles of pulsed RF plasma shown in Fig. 1 are characterized by a plateau regime with low intensities and an afterglow regime with enhanced ion signals. $\mathrm{Pt}^{-}$ions show large intensities, 
Fig. 1 a Negative mass spectrum from 16 to $830 \mathrm{Th}$ of a $\mathrm{Pt}$ foil. $\mathbf{b}$ The negative pulse profile of $\mathrm{Pt}^{-}$ions, characterized by low signals during the pulse $(2 \mathrm{~ms})$ and enhanced negative signals in the afterglow compared to the pulse on region
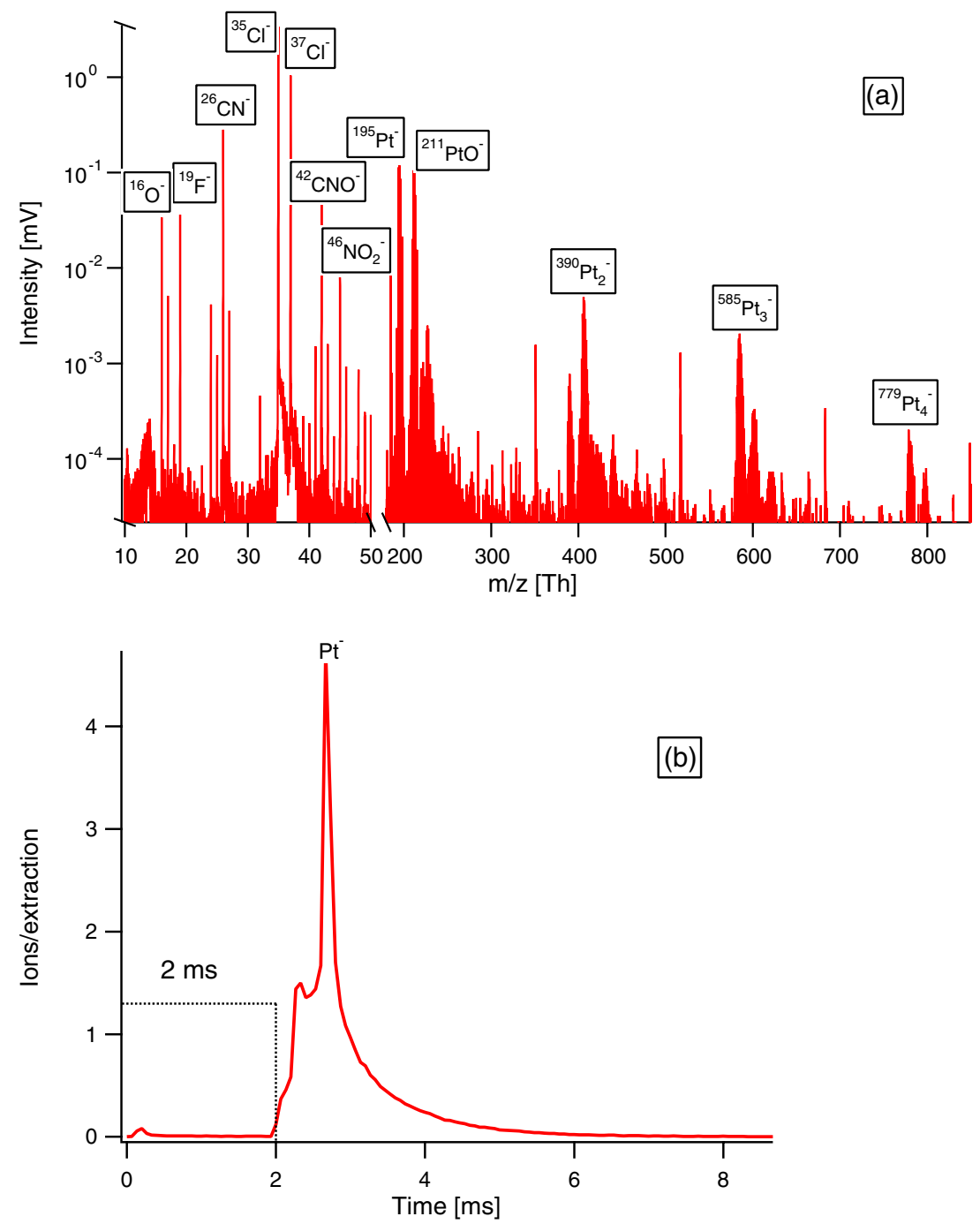

but also larger clusters, such as $\mathrm{Pt}_{2}{ }^{-}, \mathrm{Pt}_{3}{ }^{-}$, and $\mathrm{Pt}_{4}{ }^{-}$, can also be observed. The relative abundance of the cluster ions to each other and to the single platinum ions varies with time in the afterglow, but the cluster ions are always present. Pt has several isotopes, but for simplicity only the most abundant isotopes are shown in the graph. The presence of $\mathrm{PtO}^{-}$ions in the mass spectrum may indicate either a low purity of the Pt foil, or reactions with the oxygen from the background. There is a relatively abundant negative-ion background from impurities in the gas phase, such as $\mathrm{Cl}^{-}$, $\mathrm{CNO}^{-} \mathrm{NO}_{2}{ }^{-}$, and $\mathrm{NO}_{3}{ }^{-}$.

Table 1 shows the analyte signals obtained in positive and negative ion modes for several bulk materials, e.g., $\mathrm{Cu}, \mathrm{Ag}, \mathrm{Pt}$, and Au. The intensity values have been corrected for the isotopic abundance. The positive ion signals are rather similar, excepting $\mathrm{Cu}$, where the intensity is one order of magnitude lower. In case of the negative ions, the signal intensity increases with increasing electron affinity. The highest negative ion signals are thus observed for Au. It is worth mentioning that the optimum discharge conditions for metals do not vary considerably, so a direct comparison between the analyte signals of the different metals is possible. The $\mathrm{M}^{+} / \mathrm{M}^{-}$ ratios indicate that the negative ions signals are almost three orders of magnitude lower than the positive ones, so for metals, the detection of negative ions from glow discharges has little analytical utility in general.

Negative ion mode scans of several metal and metal oxides (not shown) were recorded. Our findings are summarized as follows:

1. Elements with high electron affinities, such as halogens, $\mathrm{O}, \mathrm{S}, \mathrm{Ag}, \mathrm{Pt}$, and $\mathrm{Au}$ are present as $\mathrm{M}^{-}$ions.

2. Other elements, such as $\mathrm{Si}, \mathrm{Al} \mathrm{Ti}, \mathrm{Ta}, \mathrm{Mn}$, and Fe (in an oxide matrix), were not detected as negative ions. They are mainly found as $\mathrm{MO}^{-}, \mathrm{MO}_{2}{ }^{-}, \mathrm{MO}_{3}{ }^{-}$, and $\mathrm{MO}_{4}{ }^{-}$or even larger oxides.

3. In general, it appears that larger oxide clusters are observed during sputtering of oxides in negative ion mode compared to the positive ion mode. 
Table 1 Negative analyte signals of different metals (comparison with the positive signals)

\begin{tabular}{lllll}
\hline Sample & \multicolumn{2}{l}{ Intensity $(\mathrm{cps})$} & Ratio $\left(\mathrm{M}^{+} / \mathrm{M}^{-}\right)$ & Electron affinity $(\mathrm{eV})$ \\
\cline { 2 - 3 } & Positive ions & Negative ions & & \\
\hline $\mathrm{Cu}$ & $2.55 \times 10^{6}$ & $3.6 \times 10^{2}$ & 7,075 & 1.24 \\
$\mathrm{Ag}$ & $1.02 \times 10^{7}$ & $1.5 \times 10^{3}$ & 6,800 & 1.3 \\
$\mathrm{Pt}$ & $1.6 \times 10^{7}$ & $1.5 \times 10^{4}$ & 1,000 & 1.93 \\
$\mathrm{Au}$ & $2.3 \times 10^{7}$ & $3.7 \times 10^{5}$ & 60 & 2.31 \\
\hline
\end{tabular}

Depth profiling of fluorine-containing tantala layers by GD-TOFMS

Since analytical benefits of negative ion detection are mainly given by elements with high electron affinities, negative ion GD-TOFMS depth profiling of anodic tantala samples, with known distribution of fluorine species, is now considered.

\section{Physics of fluorine ion formation}

Fluorine is not likely to be detected as a positive ion. This is primarily due to the fact that ${ }^{19} \mathrm{~F}^{+}$has an unresolved interference with ${ }^{19} \mathrm{H}_{3} \mathrm{O}^{+}\left(10^{3}-10^{4}\right.$ counts/s), which is a common gas contaminant. Ion formation from the ground state by PI is not possible because the first ionization
Fig. 2 Pulse profiles of elemental and molecular negative ions resulting from the sputtering of an anodic tantala layer containing fluorine. The RF pulse width was $500 \mu$ s. The pulse profiles were extracted from the tantala layer containing fluorine, as is it shown by the arrows in b. The $\mathrm{TaO}_{2} \mathrm{~F}^{-}$ and $\mathrm{F}^{-}$profiles denote the presence of fluorine in the tantala layer
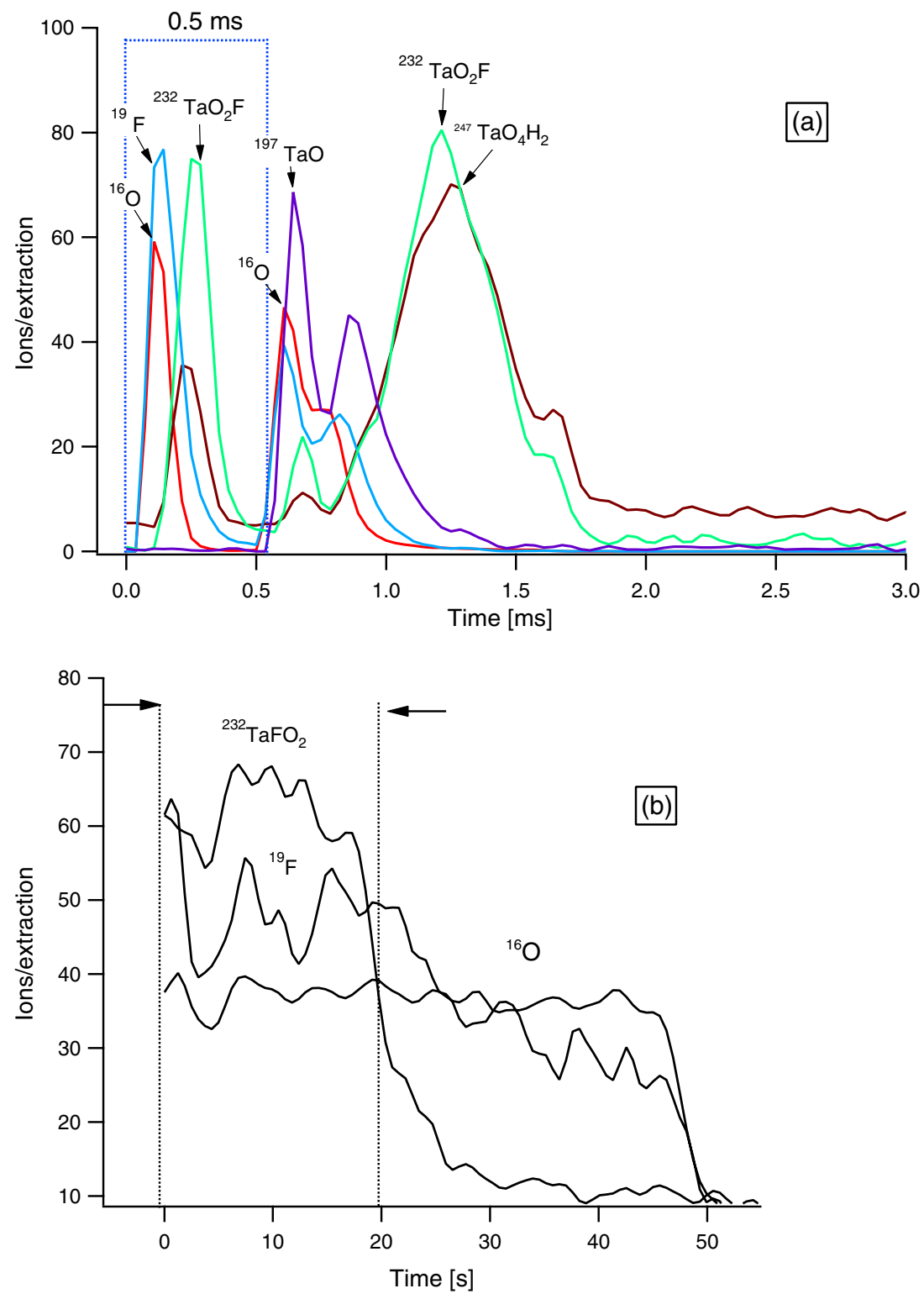
potential of $\mathrm{F}(17.32 \mathrm{eV})$ is higher than the argon metastable levels $\left({ }^{3} \mathrm{P}_{2}\right.$ at 11.55 and ${ }^{3} \mathrm{P}_{0}$ at $\left.11.72 \mathrm{eV}\right)$. However, due to its high electron affinity, fluorine negative ions can be formed in the afterglow regime through electron attachment processes.

Exciting emission lines of fluorine requires a rather large excitation energy [23], and there are no sensitive analytical lines to be selected for GD-OES analysis. Wagatsuma et al. have shown that atomic and ionic lines of $\mathrm{F}$ can be excited more efficiently in a neon plasma [24].

\section{Pulse profiles of the negative ions}

The pulse profiles of negative ions are shown in Fig. 2a. The pulse profiles were averaged over the region of the fluorine-containing layer, as it is shown by the inset diagram in Fig. 2b. A pulse width of $0.5 \mathrm{~ms}$ was found to give the largest ion signals for fluorine. In order to achieve a good depth resolution, the RF power was increased up to $60 \mathrm{~W}$. Both elemental and molecular species are characterized by pre-peak and enhanced afterglow, while the plateau region presents low intensities. The appearance of the prepeak features in the pulse profile is not clearly understood at the moment. The rise in intensity a few hundred microseconds after the termination of the RF power (first peak in the afterglow) suggests formation of negative ions by electron attachment reactions. This is in particularly true for $\mathrm{F}, \mathrm{O}$, and $\mathrm{TaO}^{-}$. In the afterglow, the electron temperature falls rapidly, and the cross section for electron attachment increases. Complex molecular ions, such as $\mathrm{TaO}_{2} \mathrm{~F}^{-}$and $\mathrm{TaO}_{4} \mathrm{H}_{2}^{-}$, appear at much later times, e.g., $700 \mu$ s after the pulse, suggesting possible negative ion formation in the flowing afterglow plasma downstream of the sampling orifice by electron capture.

\section{Depth profiling of anodic tantala films with fluorine-containing layers}

RF sputtering of the tantala films with a fluorine-containing layer results in the formation of $\mathrm{Ta}^{+}$and $\mathrm{TaO}^{+}$positive ions, while larger oxides, such as $\mathrm{TaO}_{3}^{-}, \mathrm{TaO}_{2} \mathrm{~F}^{-}$, were mainly found as negative ions. It is not clear to which extent the formation of the large oxide clusters is a consequence of the plasma chemistry, and this needs to be further investigated. GD-TOFMS depth profiles of the anodized samples were integrated from the afterglow region and are shown in Fig. 3a-c. Although the signal intensities in the pre-peak and afterglow present similar intensities, the best depth profile is obtained from the integration of the afterglow region. The oxygen profile defines the location of the anodic tantala/tantalum interface, and the $\mathrm{F}$ and $\mathrm{TaFO}_{2}$ profiles enable monitoring of the distribution of incorporated fluorine species across the anodic film thickness. In a

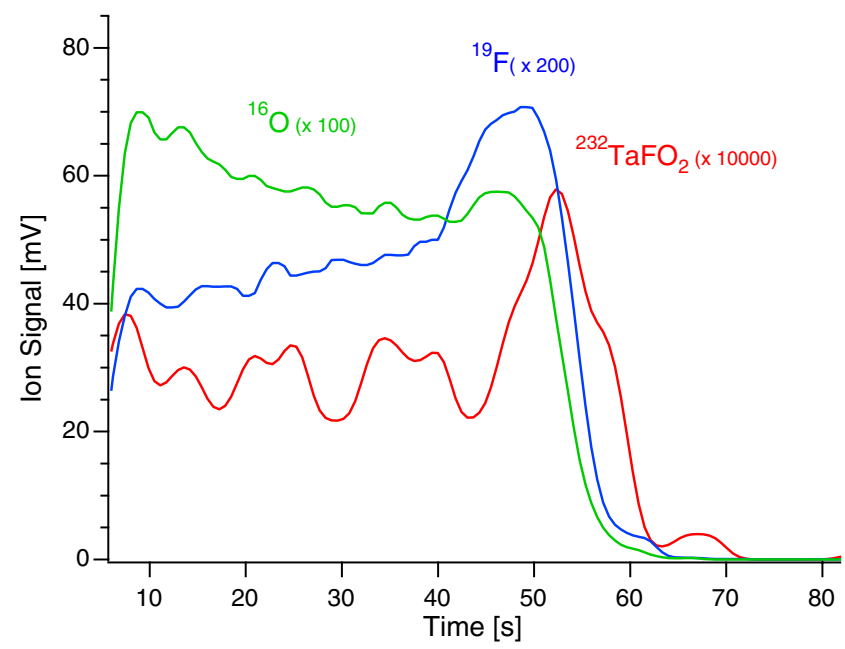

b
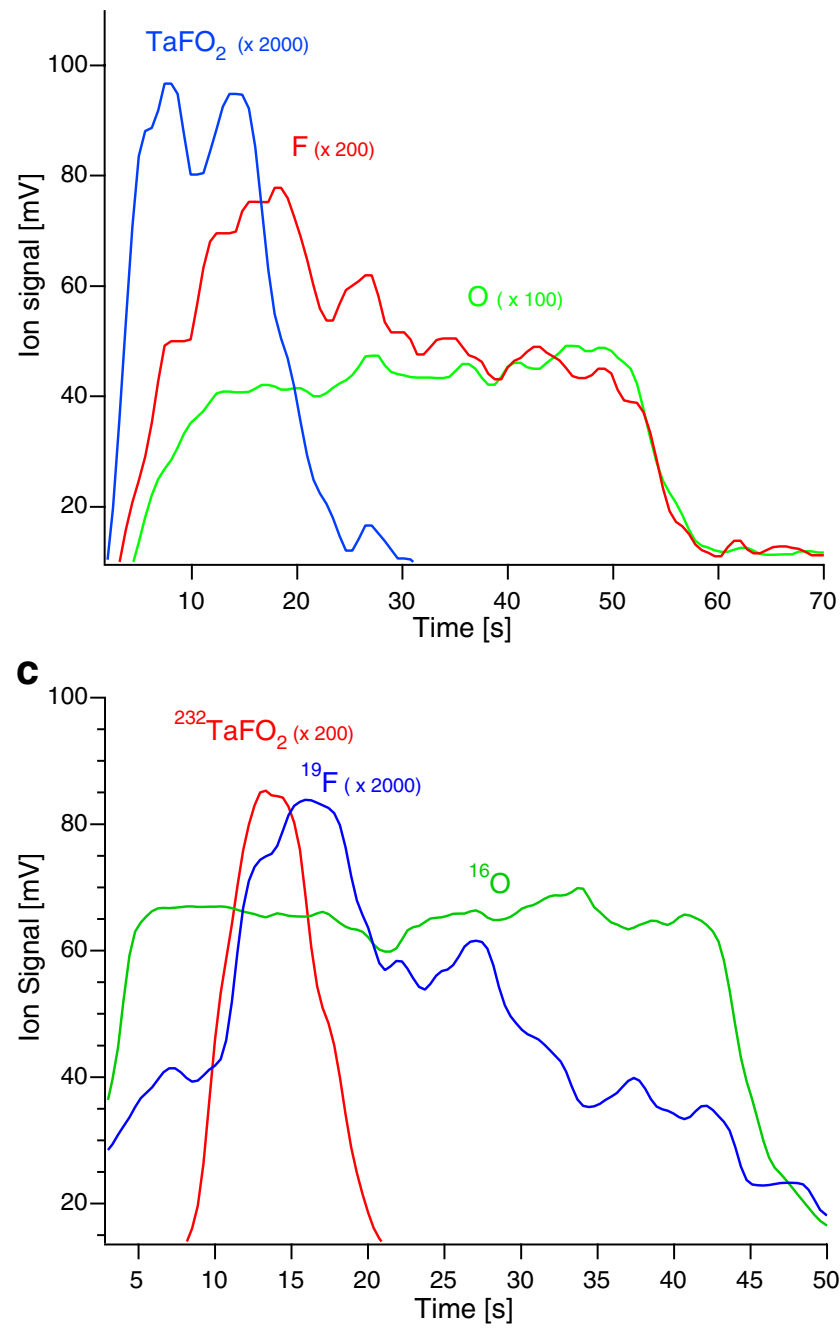

Fig. 3 GD-TOFMS depth profiles of anodic tantala films formed by sequential anodizing in APB and AF electrolytes: a specimen F100, b specimen B80-F100, c specimen B90V-F100V-B120V 
the anodic film formed by single anodizing in $\mathrm{AF}$ electrolyte (Fig. 3a), a relatively uniform distribution of fluorine species is evident through most of the anodic tantala film thickness, with an increased yield of fluorine species in the film region immediately adjacent to the tantalum substrate. The presence of increased fluoride yield in the narrow, near-interface region confirms that inward migration of $\mathrm{F}^{-}$ions is faster than $\mathrm{O}^{2-}$ ions during formation of the anodic tantala film. According to a previous XPS study, the fluorine-containing layer adjacent to the tantalum substrate is $\mathrm{TaF}_{5}$ [18]. The anodic film formed by sequential anodizing in APB and AF electrolytes reveals the presence of fluoride-containing outer film region (Fig. 3b). Finally, sequential anodizing in APB, AF, and APB electrolytes results in formation of a fluoridecontaining layer within the thickness of the anodic tantala film (Fig. 3c).

At high current efficiency, growth of anodic tantala films proceeds at the anodic tantala/tantalum and anodic tantala/ electrolyte interfaces through inward and outward movement of $\mathrm{O}^{2-}$ and $\mathrm{Ta}^{5+}$ ions, respectively. The voltage-time response recorded during formation of anodic film of sample B80V-F100V discloses an initial voltage surge of about $2 \mathrm{~V}$ at the commencement of anodizing, indicating the presence of a thin, 2-3-nm-thick, air-formed oxide film over the macroscopic tantalum surface (Fig. 4a). Subsequently, the voltage increases linearly with anodizing time with of $0.38 \mathrm{~V} / \mathrm{s}$ for anodizing in APB electrolyte. After changing electrolyte for $\mathrm{AF}$, a short transition region of rapidly increasing voltage is evident. When the voltage reaches approximately $80 \mathrm{~V}$, anodizing proceeds with a linearly increasing voltage, but with a reduced slope of $0.28 \mathrm{~V} / \mathrm{s}$. Taking into account that formation of anodic tantala film in APB electrolyte proceeds at approximately $100 \%$ efficiency, the reduced rate of voltage rise during film growth in AF suggests a decrease of current efficiency. Dividing the slopes obtained during anodizing in APB and AF electrolytes suggests a current efficiency for anodizing in $\mathrm{AF}$ electrolyte of about $73 \%$. Given the transport numbers of $\mathrm{Ta}^{5+}$ and $\mathrm{O}^{2-}$ ions of 0.24 and 0.76 , then $24 \%$ and $76 \%$ of the total current is consumed for film growth at the electrolyte/anodic film and anodic film/tantalum interfaces, respectively, at $100 \%$ current efficiency. The reduced current efficiency of $73 \%$ for anodizing in fluoride electrolyte suggests that all outwardly moving $\mathrm{Ta}^{5+}$ ions are ejected in the electrolyte, with film growth then proceeding only at the anodic tantala/tantalum interface. The current efficiency of $73 \%$ is $3 \%$ less than that
Fig. 4 Film formation on tantalum under conditions employed for specimen B80-F100: a voltage-time response recorded during anodizing; schematic diagrams showing the layered structure and extents of migration of species after $\mathbf{b}$ anodizing in APB and $\mathbf{c}$ re-anodizing in $\mathrm{AF}$ electrolyte
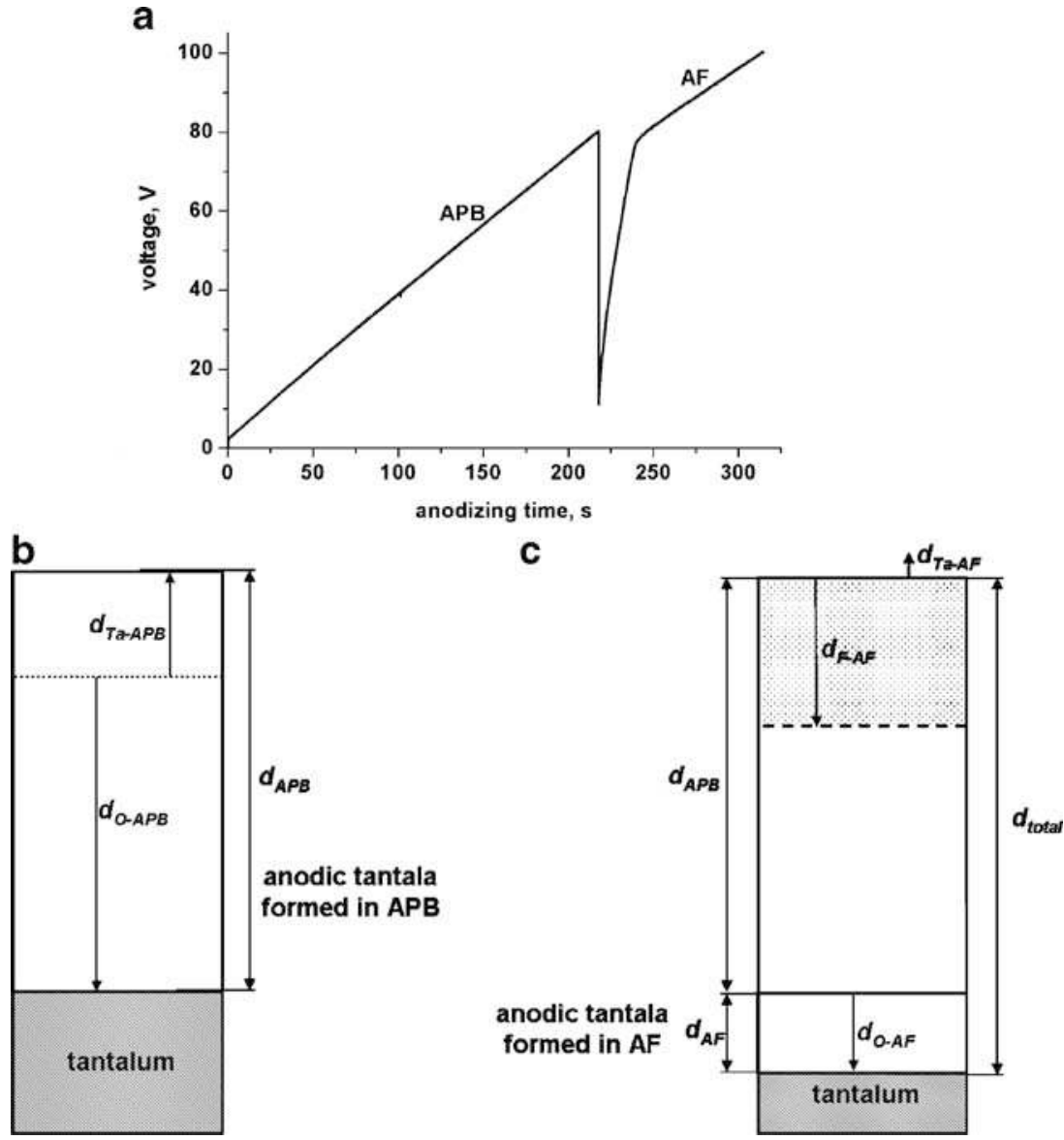
anticipated for film formation only at the anodic film/ tantalum interface, i.e., $76 \%$. Hence, the small difference in efficiency is considered to be due to experimental error.

Transport processes during anodic film growth also involve incorporation of species derived from the electrolyte. Given the transport numbers of $\mathrm{O}^{2-}$ and $\mathrm{Ta}^{5+}$ ions and the mobilities of electrolyte-derived species, their distributions may be predicted precisely. The rate of migration of fluoride ions may be calculated from the depth profiles of Fig. $3 \mathrm{~b}, \mathrm{c}$. For simplicity, the depth profile of the anodic film formed by anodizing in APB and re-anodizing in AF electrolyte (Fig. 3b) is considered. Schematic diagrams of the layered anodic film and associated ionic migrations involved during film growth are illustrated in Fig. 4b, c, with Fig. $4 \mathrm{~b}$ displaying the anodic film formed in APB electrolyte. The distances $d_{\mathrm{Ta}-\mathrm{APB}}$ and $d_{\mathrm{O}-\mathrm{APB}}$ denote the extents of migration of $\mathrm{Ta}^{5+}$ and $\mathrm{O}^{2-}$ ions relative to the original sample surface, i.e., the imaginary dotted line and, correspondingly, the thicknesses of film regions formed at the electrolyte/anodic film and anodic film/tantalum interfaces. In accordance with transport numbers, the relative thicknesses of the regions may be expressed as $d_{\mathrm{Ta}-\mathrm{APB}} / d_{\mathrm{O}-\mathrm{APB}}=0.24 / 0.76$. Further, re-anodizing in $\mathrm{AF}$ fluoride electrolyte (Fig. 4c) results in growth of the anodic film only at the anodic film/tantalum interface, with $\mathrm{Ta}^{5+}$ ions moving outward, but ejected to the electrolyte without forming film material. The thickness of the film added by re-anodizing is determined by the distance passed by $\mathrm{O}^{2-}$ ions, $d_{\mathrm{O}-\mathrm{AF}}$. From the nanometer-to-voltage formation ratio of anodic tantala film, $1.62 \mathrm{~nm} / \mathrm{V}$, at a current density of $1 \mathrm{~mA} / \mathrm{cm}^{2}$, the thickness of the film formed in APB $d_{\mathrm{APB}}$ is $80 \mathrm{~V} \times 1.62 \mathrm{~nm} / V=129.6 \mathrm{~nm}$. The calculated thickness of film region formed in $\mathrm{AF} d_{\mathrm{AF}}=d_{\mathrm{O}-\mathrm{AF}}$ is $20 \mathrm{~V} \times 1.62 \mathrm{~nm} / \mathrm{V}=32.4 \mathrm{~nm}$, giving a total film thickness generated by sequential anodizing in APB and AF electrolytes, $d_{\text {total }}$, of $162 \mathrm{~nm}$. Assuming that the sputtering rate of the anodic tantala film of $2.96 \mathrm{~nm} / \mathrm{s}$ is constant across the film thickness, then the thickness of the outer fluoridecontaining region, $d_{\mathrm{F}-\mathrm{AF}}$, measured from the depth profiles of Fig. 3b, is approximately $50.3 \mathrm{~nm}$. The mobility of $\mathrm{F}^{-}$ ions, determined as $d_{\mathrm{F}-\mathrm{AF}} / d_{\mathrm{O}-\mathrm{AF}}$, is about 1.6 ; thus, during film growth, fluoride ions migrate inward about 1.6 times as fast as oxygen ions.

GD-OES analysis of the samples, using neon plasma, revealed similar distributions of fluorine species within the anodic tantala films (Fig. 5a-c). Waviness of the tantalum profiles, resulting from optical interference in the optically transparent material, is evident. Further, the response from fluorine species appears noisier compared with the response from $\mathrm{TaFO}_{2}$ in GD-TOFMS examination, suggesting a higher sensitivity of GD-TOFMS in the negative mode for detection of fluorine. The mobility of fluorine ions, calculated from depth profile of Fig. 5b, is about 1.6 that

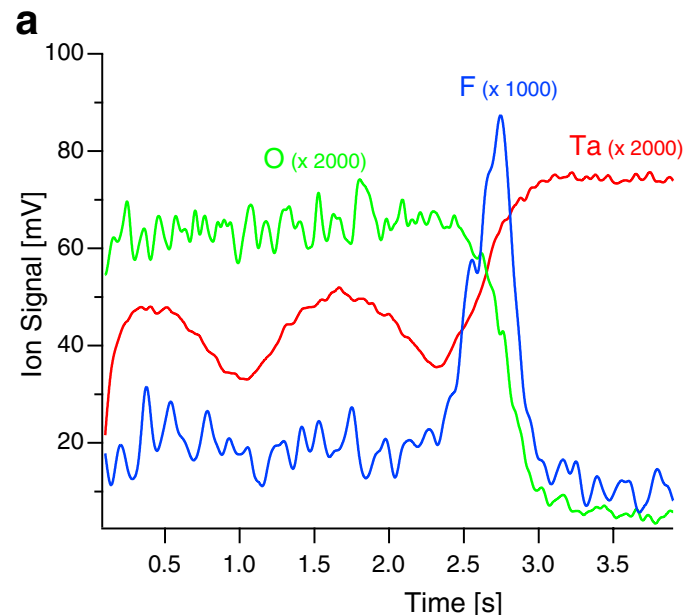

b

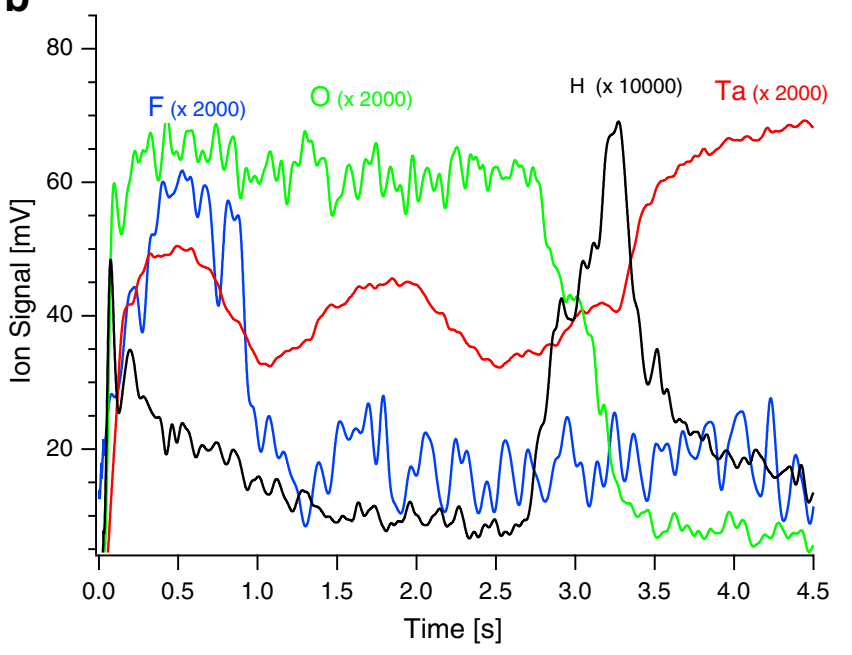

C

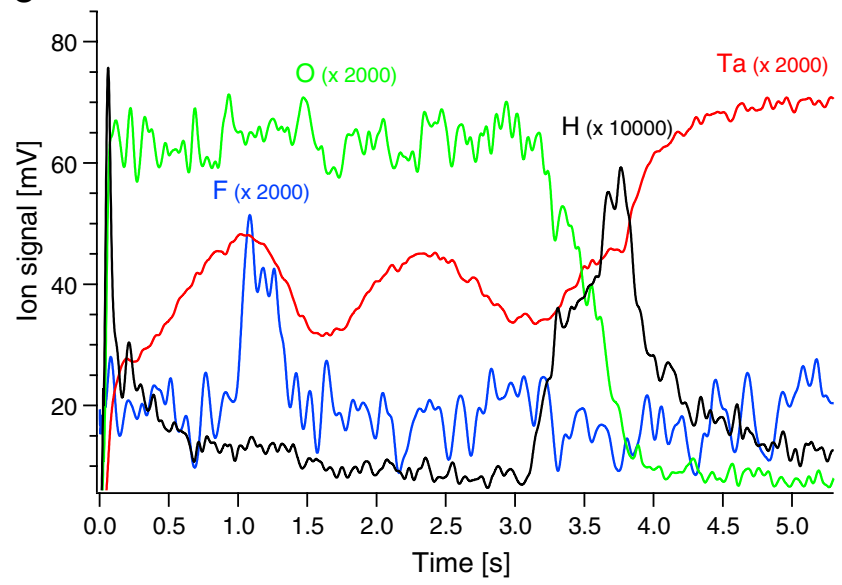

Fig. 5 GD-OES depth profiles of anodic tantala films formed by sequential anodizing in APB and AF electrolytes obtained in neon plasma: a specimen F100, b specimen B80-F100, c specimen B90VF100V-B120V

confirms that obtained by GD-TOFMS. The obtained value is, however, less than that of 2 obtained previously from SIMS analysis [18]. The possible explanation may be associated with the presence of relatively intense hydrogen 
peak near the anodic film/tantalum interface. Hydrogen may affect significantly glow discharge parameters, with consequent change of line intensities, sputtering rates, and discharge currents $[25,26]$. The change of sputtering rate near the interface may correspondingly reduce the accuracy of measurements, with consequent difference when compared with SIMS data.

\section{Conclusions}

The data presented here demonstrate that negative ion detection mode can be successful employed to study distribution of fluorine layers in anodic tantala or others in glow discharge mass spectrometry. Detecting fluorine by negative ion GD-TOFMS is an elegant and rapid analysis technique compared to GD-OES, where neon plasma is commonly used to excite fluorine atoms. The analytical utility of the negative ion must still be explored for other complex materials, such as polymers, etc.

Acknowledgment Financial support from the European Union in the form of a Framework 6 STREP grant for the "New Elemental and Molecular Depth Profiling Analysis of Advanced Materials by Modulated Radio Frequency Glow Discharge Time of Flight Mass Spectrometry" (EMDPA) project is also gratefully acknowledged.

\section{References}

1. Nickel H, Broekaert JAC (1999) Fresenius' J Anal Chem 363:145-155

2. Pisonereo J, Fernández B, Günther D (2009) J Anal At Spectrom 24:1145-1160
3. Fulford JE, Quan ESK (1988) Appl Spectrosc 42:425-428

4. Oechsner H (1995) Int J Mass Spectrom Ion Process 143:271-282

5. Hoffmann V, Kasik M, Robinson PK, Venzago C (2005) Anal Bioanal Chem 381:173-188

6. Hohl M, Kanzari A, Michler J, Nelis T et al (2006) Surf Interface Anal 38:292-295

7. Weiss Z, Marshall K (1997) Thin Solid Films 308-309:382-388

8. Pisonero J (2006) Anal Bioanal Chem 384:47-49

9. Muniz AC, Pisonero J, Lobo L, Gonzalez C et al (2008) J Anal At Spectrom 23:1239-1246

10. Tuccitto N, Lobo L, Tempez A, Delfanti I et al (2009) Rapid Commun Mass Spectrom 23:549-556

11. Bogaerts A, Gijbels R (1998) Spectrochim Acta Part B 53:1-42

12. Pan C, King FL (1993) J Am Soc Mass Spectrom 4:727-732

13. King FL, Pan C (1993) Anal Chem 65:735-739

14. Vickers GH, Wilson DA, Hieftje GM (2002) Anal Chem 60:1808-1812

15. Bentz BL, Harrison WW (1981) Int J Mass Spectrom Ion Phys $37: 167-176$

16. Canulescu S, Whitby J, Fuhrer K, Hohl M et al (2009) J Anal At Spectrom 24:178-180

17. Tempez A, Canulescu S, Molchan IS, Döbeli M et al (2009) Surf Int Anal 41(12-13):966-973

18. Shimizu K, Kobayashi K, Thompson GE, Skeldon P et al (1996) Philos Mag, B 73:461-485

19. Pisonero J, Feldmann I, Bordel N, Sanz-Medel A et al (2005) Anal Bioanal Chem 382:1965-1974

20. Molchan IS, Thompson GE, Skeldon P, Trigoulet N et al (2009) J Anal At Spectrom 24:734-741

21. Stoffels E, Stoffels WW, Vender D, Haverlag M et al (1995) Contrib Plasma Phys 35:331-357

22. Overzet LJ, Lin Y, Luo L (1992) J Appl Phys 72:5579-5592

23. Wagatsuma K, Hirokawa K, Yamashita N (1996) Anal Chim Acta 324:147-154

24. Habazaki H, Fushimi K, Shimizu K, Skeldon P et al (2007) Electrochem Commun 9:1222-1227

25. Martín A, Menéndez A, Pereiro R, Bordel N et al (2007) Anal Bioanal Chem 388:1573-1582

26. Hodoroaba V-D, Hoffmann V, Steers EBM, Wetzig K (2000) J Anal At Spectrom 15:1075-1080 